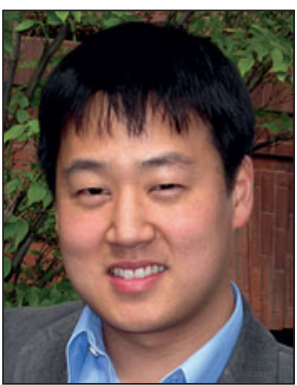

Minjoo Larry Lee

Guest Editor for this issue of MRS Bulletin

Department of Electrical Engineering,

Yale University, USA; tel. 203-432-4298; and

email minjoo.lee@yale.edu or

mllee@alum.mit.edu.

Lee joined the Department of Electrical Engineering at Yale University in 2008. He received his ScB degree from Brown University in 1998, and his $\mathrm{PhD}$ degree in electronic materials from Massachusetts Institute of Technology (MIT) in 2003. From 2003 to 2006, he was a postdoctoral researcher with the Microsystems Technology Laboratory at MIT, and from 2006 to 2007, he was with the Center for Thermoelectrics Research at RTI International in Durham, N.C. He is the author or co-author of over 100 technical papers and conference proceedings and holds nine patents. Lee's recognitions include the North American Molecular Beam Epitaxy Conference Young Investigator Award, DARPA Young Faculty Award, National Science Foundation CAREER Award, and the IEEE Electron Device Society George E. Smith Award.

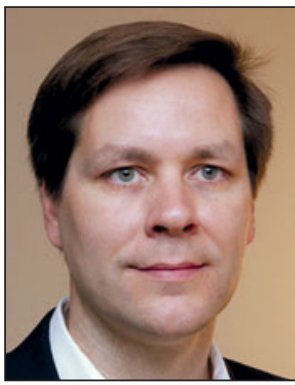

Christopher J.K. Richardson

Guest Editor for this issue of MRS Bulletin

Laboratory for Physical Sciences, University of Maryland, USA; tel. 301-935-6400;

and email richardson@Ips.umd.edu.

Richardson is a research scientist at the Laboratory for Physical Sciences at the University of Maryland. He received his BS degree in engineering physics from the University of Maine, and $\mathrm{MS}$ and $\mathrm{PhD}$ degrees in materials science and engineering from Johns Hopkins University. He has authored papers and conference contributions in technical areas spanning epitaxial material design, growth, materials characterization, and optoelectronic devices. Richardson's current research interests include molecular beam epitaxy of dissimilar materials and the materials science of quantum computing.

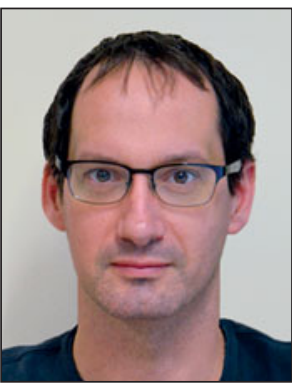

Laurent Cerutti

Université de Montpellier, France;

tel. +33-467143281; and email

laurent.cerutti@umontpellier.fr.

Cerutti became an associate professor at the Université de Montpellier, France, in 2006, where he received his $\mathrm{PhD}$ degree in optoelectronics. From 2004 to 2006, he was at the Technical University of Madrid, Spain, as a postdoctoral fellow developing a growth method using molecular beam epitaxy for GaN nanocolumns on $\mathrm{Si}(100)$. Cerutti's research focuses on the growth and study of Sb-based optoelectronic devices.

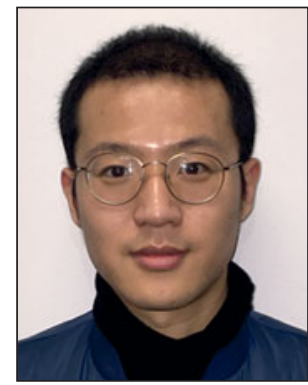

Siming Chen

Department of Electronic and Electrical Engineering, University College London, UK; tel. +44 (0)20 7679 0178; and email siming.chen@ucl.ac.uk.

Chen joined the Department of Electronic and Electrical Engineering at University College London, UK in 2013 as a research associate. He received his $\mathrm{PhD}$ degree in electrical engineering from The University of Sheffield in 2014 $\mathrm{He}$ has been working on III-V and Si-based quantum dot optoelectronics devices and silicon photonics.

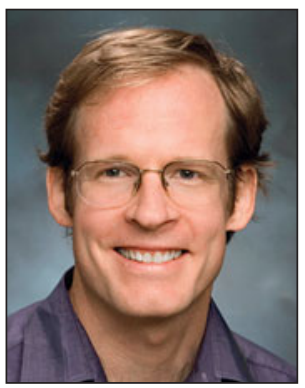

Peter Deelman

HRL Laboratories, LLC, USA; tel. 310-317-5843; and email pwdeelman@hrl.com.

Deelman is a senior research staff engineer at HRL Laboratories, LLC. He received his PhD degree in physics from Rensselaer Polytechnic Institute. Prior to joining HRL, he worked at the Jet Propulsion Laboratory in Pasadena, Calif. His research interests include the growth and fabrication of III-V and SiGe heteroepitaxial structures.

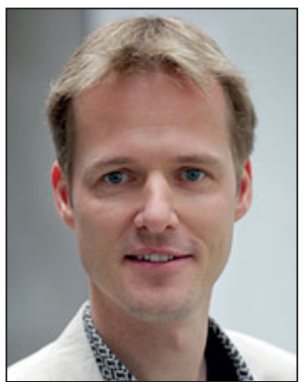

\section{Frank Dimroth}

Division Materials-Solar Cells and Technology, Fraunhofer Institute for Solar Energy Systems, Germany; tel. +49 (0)761/4588-5258; and email frank.dimroth@ise.fraunhofer.de. Dimroth heads the III-V Epitaxy and Solar Cells Department at the Fraunhofer Institute for Solar Energy Systems. This group carries out applied research in the fields of III-V multijunction solar cells and concentrator photovoltaic systems, and developed record solar-cell devices with efficiency up to $46 \%$ at 500 suns. He received his diploma in physics from the University of Zurich in 1996, and his PhD degree in physics from the University of Constance in 2000. He and his team received the Joseph von Fraunhofer Prize in 2010, the French "Fondation Louis D" Award in 2010, and the French-German Economy Prize in 2011. Dimroth has more than 150 publications and serves as editor for the IEEE Journal of Photovoltaics in the field of III-V, Concentrator, and Space PV.

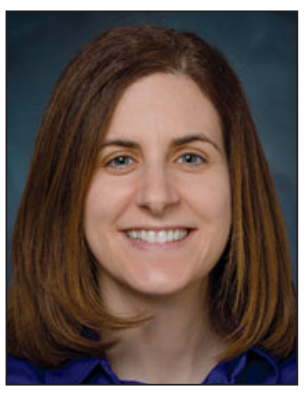

Lisa Edge

HRL Laboratories, LLC, USA; tel. 310-317-5907; and email lfedge@hrl.com.

Edge is a senior research staff scientist at HRL Laboratories, LLC. She received her PhD degree in materials science and engineering from The Pennsylvania State University. Prior to joining HRL, she worked for eight years at IBM in Albany, N.Y. Edge's research interests include the epitaxial growth of materials, and the interactions and surface science between these epitaxial materials and high- $k$ gate dielectrics.

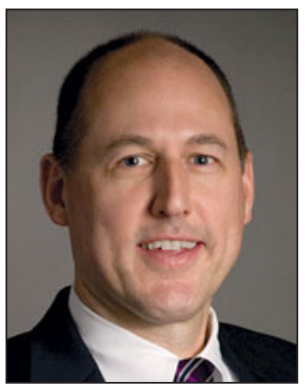

\section{Eugene A. Fitzgerald}

Department of Materials Science and

Engineering, Massachusetts Institute of Technology, and Singapore-Massachusetts Institute of Technology Alliance for Research and Technology, USA; tel. 617-258-7461; and email eafitz@mit.edu.

Fitzgerald is the Merton C. Flemings SMA Professor of Materials Science and Engineering at the Massachusetts Institute of Technology (MIT) and Lead Principal Investigator of the Low Energy Electronic Systems Center of the Singapore-MIT Alliance for Research and Technology. He received his SB degree from MIT in 1985, and his PhD degree from Cornell University in 1989, both in materials science. His research interests include novel thin-film materials and devices. He is the co-inventor of high-mobility strained silicon, and has founded several startups. Fitzgerald received the IEEE 2011 Andrew S. Grove Award and the IEEE 2004 EDS George Smith Award. 


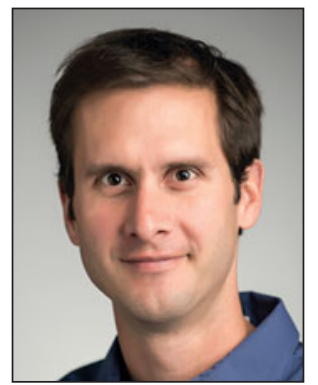

Ryan France

National Renewable Energy Laboratory, USA; tel. 303-384-6441; and email

ryan.france@nrel.gov.

France is a scientist in the III-V Multijunctions Group at the National Renewable Energy Laboratory (NREL), where he researches metamorphic epitaxy, lattice-mismatched solar cells, and the development of new absorber materials for multijunction solar cells. He received his bachelor's degree in applied physics from Washington University in St. Louis, his master's degree in electrical engineering from Boston University, and his PhD degree in materials science at the Colorado School of Mines. France has studied the interaction between atomic ordering and dislocations in III-V materials, and helped develop high-efficiency three- and four-junction inverted metamorphic multijunction devices.

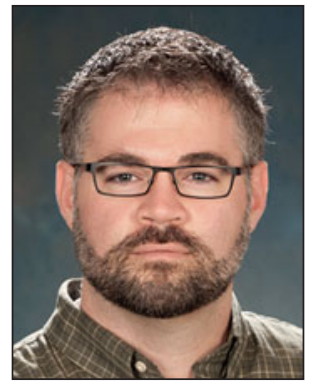

\section{Tyler J. Grassman}

Department of Materials Science and Engineering, and Department of Electrical and Computer Engineering, The Ohio State University, USA; tel. 614-688-1704; and email grassman.5@osu.edu.

Grassman is an assistant professor at The Ohio State University, with joint appointments in the Departments of Materials Science and Engineering, and Electrical and Computer Engineering. He received his $\mathrm{BA}$ degree in chemistry at the University of Oregon in 2000, and his PhD degree in materials science and engineering at the University of California, San Diego in 2007. Grassman's research interests focus on the epitaxy and characterization of novel and integrated materials for optoelectronics applications, with a particular focus on photovoltaics.

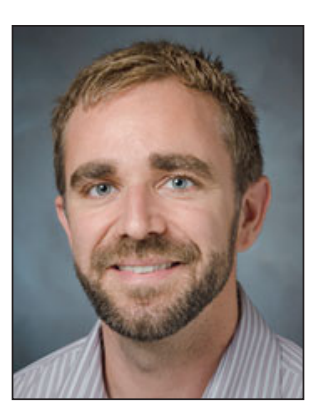

\section{Clayton Jackson}

HRL Laboratories, LLC, USA; tel. 310-317-5530; and email cajackson@hrl.com.

Jackson is a research staff member at HRL Laboratories, LLC. He received his PhD degree in materials engineering from the University of California, Santa Barbara. His research interests include the growth and characterization of lowdimensional semiconductor heterostructures.

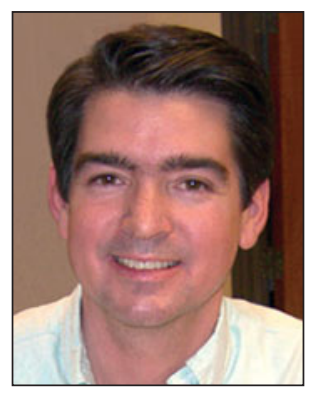

\section{Richard R. King}

School of Electrical, Computer, and

Energy Engineering, Arizona State University, USA; tel. 480-727-3698; and

email richard.r.king@asu.edu.

King is a professor in the School of Electrical, Computer, and Energy Engineering at Arizona State University. He received his BS degree in physics and MS and PhD degrees in electrical engineering, all from Stanford University. His research has explored metamorphic III-V materials, high-lifetime back-contact silicon solar cells, dilute nitride GalnNAs(Sb), ordered and disordered (Al)GalnP, recombination in compound semiconductors, and high-efficiency multijunction solar cells. He is the recipient of the 2010 William R. Cherry Award given by the IEEE. King is a co-founding editor of the IEEE Journal of Photovoltaics, and served as general chair for the 40th IEEE Photovoltaic Specialists Conference in 2014.

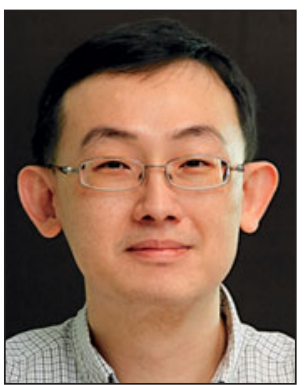

Kenneth E. Lee

Singapore-Massachusetts Institute of Technology Alliance for Research and Technology, Singapore; tel. +65-6601-3209; and email kenneth.lee@smart.mit.edu.

Lee is the scientific director of the Low Energy Electronic Systems Center of the SingaporeMassachusetts Institute of Technology (MIT) Alliance for Research and Technology. Prior to this, he worked in Singapore's Ministry of Defence Temasek Laboratories at Nanyang Technological University, and DSO National Laboratories. Lee received his BS and MS degrees from the University of Illinois at Urbana-Champaign in 1998 and 1999, respectively, and his $\mathrm{PhD}$ degree from MIT in 2009, all in electrical engineering.

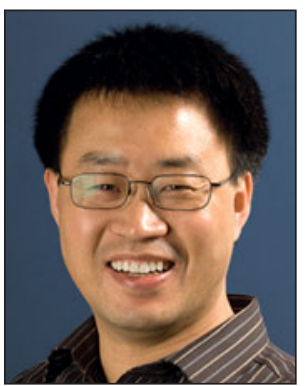

\section{Huiyun Liu}

Department of Electronic and Electrical Engineering, University College London, UK; tel. +44 (0)20 7679 3983; and email huiyun.liu@ucl.ac.uk.

Liu is professor of semiconductor photonics in the Department of Electronic and Electrical Engineering at University College London (UCL) He received his $\mathrm{PhD}$ degree in semiconductor science from the Institute of Semiconductors, Chinese Academy of Sciences, in 2001. He joined UCL in 2007. Liu's current research interest focuses on the nanometer-scale engineering of low-dimensional semiconductor structures, such as quantum dots and nanowires for laser and solar cells.

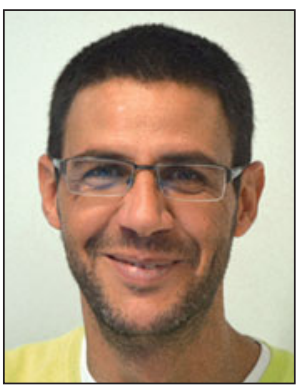

Jean-Baptiste Rodriguez

Université de Montpellier, France;

tel. +33-467143281; and email

jean-baptiste.rodriguez@umontpellier.fr.

Rodriguez obtained his PhD degree from the Université de Montpellier, France, in 2005. After a two-year postdoctorate at the Center for High Technology Materials at The University of New Mexico, he joined the Institute of Education Sciences in 2008 as a CNRS researcher. His interests include the strain relaxation of antimonidebased materials on highly mismatched substrates, and development of InAs/GaSb SL photodetectors. He is the author or co-author of approximately 80 articles in peer-reviewed journals and conference proceedings, one book chapter, and one international patent.

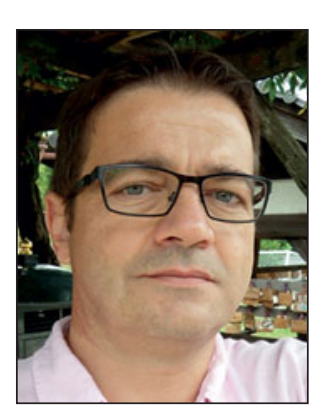

\section{Eric Tournié}

Université de Montpellier, and Institut Universitaire de France, France; tel. +33-467143280; and email eric.tournie@umontpellier.fr.

Tournié was appointed as a professor of electrical engineering by the Université de Montpellier in 2002. He received his $\mathrm{PhD}$ degree in optoelectronics from the University of Montpellier in 1990. He then joined the Max Planck Institute in Stuttgart, Germany, as a postdoctoral scientist. In 1993, he spent a few months with the Paul Drude Institute in Berlin, Germany, before joining CRHEA-CNRS, France. Tournié is working on the development of GaSbbased infrared devices, including their integration on silicon platform. 


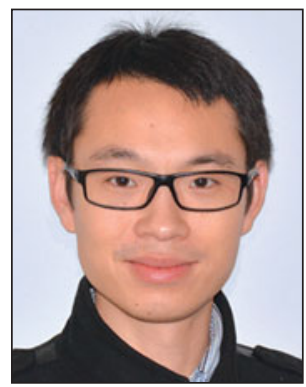

Jiang Wu

Department of Electronic and Electrical Engineering, University College London, UK; tel. +44 (0)20 7679 0178; and email jiang.wu@ucl.ac.uk.

Wu has been a lecturer in the Department of Electronic and Electrical Engineering at University College London (UCL) since 2015. He received his $\mathrm{PhD}$ degree in electrical engineering from the University of Arkansas in 2011. In 2012, he joined the photonics group at UCL as a research associate prior to his present position. Wu has been working on molecular beam epitaxy of III-V semiconductors and quantum dot optoelectronic devices.
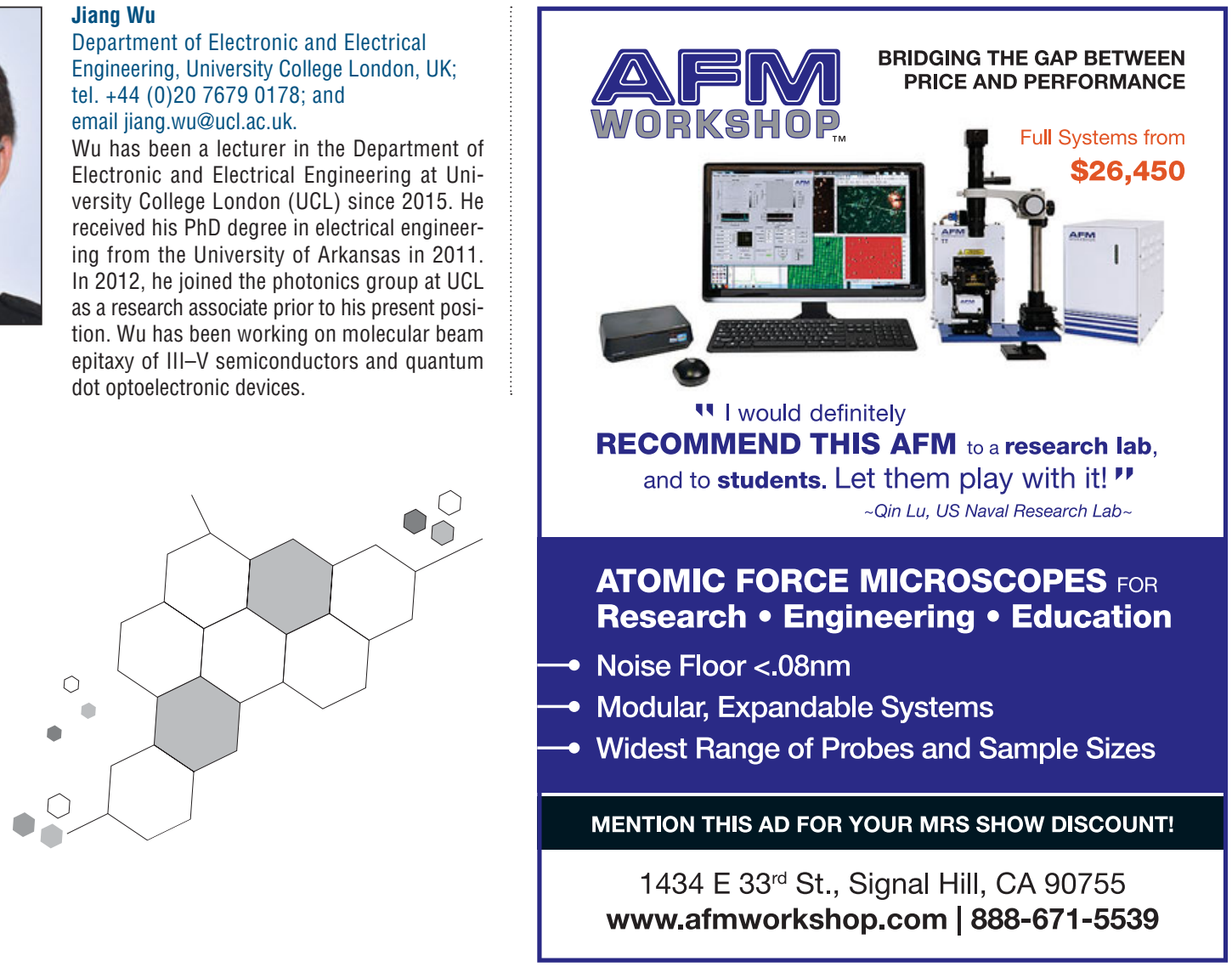

\section{(2) Rigaku Leading With Innovation

Rigaku Miniflex300/600 can be equipped with the D/teX Ultra high-speed 1-dimensional detector to obtain greater intensity. This detector has a broad detection surface and can efficiently count diffraction $X$-rays from a sample. As a result, it is possible to obtain intensities from a few tens to roughly 100 times greater than a scintillation counter. Background can also be reduced using the fluorescent $X$-ray reduction mode or the knife edge feature. The figure shows the $X$-ray diffraction patterns obtained when a knife edge is installed versus no knife edge. By using a knife edge, it is possible to achieve an extreme reduction in scattering on the low-angle side. Also, the knife edge moves in a way which is dependent on the diffraction angle $2 \theta$, and thus there is no blind spot (intensity attenuation) on the high-angle side, and measurement can be done from the low-angle side to the high-angle side.

\section{Miniflex}

$X$-ray diffraction patterns (enlarged) of zeolite, measured when a knife edge is installed versus no knife edge.

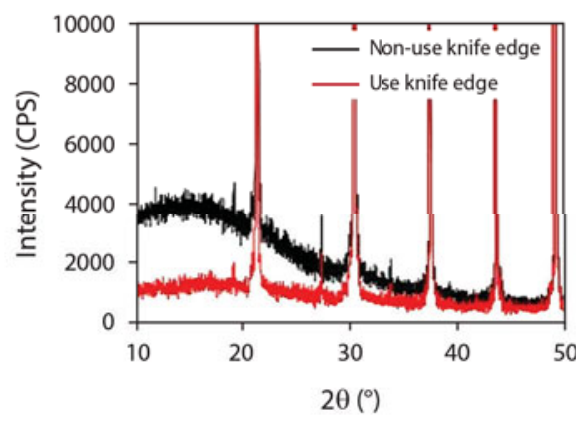

Rigaku Corporation and its Global Subsidiaries www.Rigaku.com | info@Rigaku.com

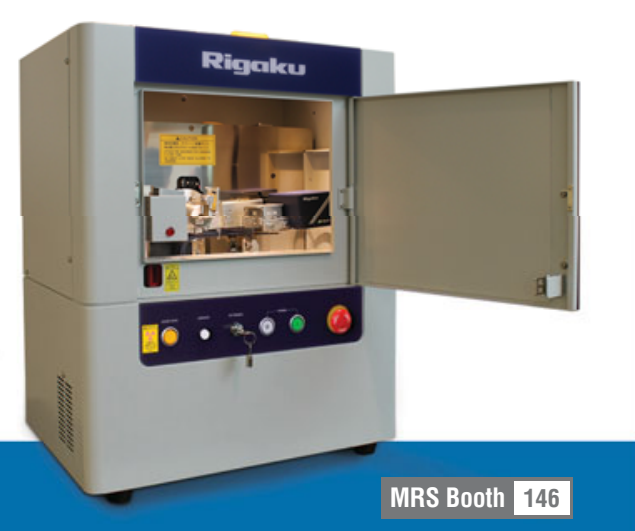

